\title{
Abscopal effect in head and neck cancer: a unicorn summoned once every eon?
}

\begin{abstract}
The introduction of immune checkpoint inhibitors (ICI), such as anti-PD1, anti-PDL1 and anti-CTLA4, has shown clinically significant benefit in prospective randomized clinical trials across many tumor types. In recurrent/metastatic head and neck squamous cell cancer ICIs have overall response rates $\sim 13-18 \%$. The realization that radiotherapy may induce outof-field immune-related effects, known as abscopal, by acting as an "in-situ" vaccine has led the research to combined radioimmunotherapy studies. In this short review we follow the abscopal effect from its first case report to the present and contemplate on how the delivery of radiotherapy could be optimized to maximize the probability of its occurrence.
\end{abstract}

Keywords: head and neck cancer, abscopal effect, tumor lesions, laryngeal cancer
Volume 10 Issue 5 - 2019

\author{
Efstathios Kamperis,' Chionia Kodona, ${ }^{2}$ \\ Konstantinos Markou, ${ }^{3}$ Vasileios \\ Giannouzakos' \\ 'Department of Radiation Oncology, Papageorgiou General \\ Hospital, Greece \\ 2Department of Medical Physics, Papageorgiou General Hospital, \\ Greece \\ ${ }^{3}$ Department of ENT,Aristotle University of Thessaloniki, \\ Papageorgiou General Hospital, Greece
}

\begin{abstract}
Correspondence: Efstathios Kamperis, Department of Radiation Oncology, Papageorgiou General Hospital, Agiou Pavlou 76, PO 564 29, Thessaloniki, Greece, Tel 231332 3417 Email ekamperi@gmail.com
\end{abstract}

Received: October 06, 2019 | Published: October 18, 2019
Abbreviations: TMB, tumor mutational burden; DAMP, damage-associated molecular patterns; DC, dendritic cells; BED, biological effective dose; SBRT, stereotactic body radiotherapy

\section{Introduction}

Radiotherapy has a decades-long history of use as an effective local treatment in the management of cancer. Its therapeutic effect is commonly explained on the basis of direct and indirect DNA damage to rapidly proliferating cancer cells. However, mounting preclinical and clinical evidence suggest that radiation is capable of inducing tumor-specific immune-mediated responses. ${ }^{1}$

Abscopal effect is the reduction of tumor lesions located outside the irradiation field, assuming no other active systemic cytotoxic is administered concurrently. The term was first coined by Mole and etymologically is derived from the Latin prefix "ab" which means "position away from" and the word "scopos" which means "target". ${ }^{2}$ Hence, abscopal effect is an "effect away from the target". It is noteworthy that Mole used the term to describe radiation effects to normal organs, not tumors. In specific, he noticed that thyroid depression would appear only when sufficient volume of the abdomen was irradiated. Although the term was introduced in the middle of the 20th century, the first clinical case is often attributed to McCulloch who, in 1908, irradiated the regional lymph nodes of a patient with locally advanced unresectable laryngeal cancer achieving complete remission. $^{3}$

The second clinical case of abscopal effect in head and neck cancer (or the first, since McCulloch's patient technically had regional disease, not distant metastatic) was reported by Shinde et al. ${ }^{4} 111$ years later. It refers to a patient with newly diagnosed locally advanced oropharyngeal and hypopharyngeal cancer with de novo lung metastases. The patient was enrolled in a clinical trial comparing the EXTREME regimen (cisplatin/5FU/cetuximab) vs. ipilimumab and nivolumab. He was randomized to the combined immunotherapy arm and during the first follow-up, after having received one cycle, progressive disease was documented. The primary tumor enlarged and both the size and the number of lung metastases increased eliminating the possibility of pseudo progression. The patient underwent palliative radiotherapy in the neck with the "QUAD" shot regimen (2 fractions per day of 3.7 Gy per fraction for 2 days). During restaging, a $25 \%$ reduction of the primary tumor was documented along with a $50 \%$ reduction in lung metastases. The patient continued receiving more "QUAD" shot courses and 10 months later he has a stable disease.

\section{Immunotherapy in head and neck cancer}

Head and neck cancer is characterized by a relatively high prevalence of somatic mutations and regular formation of neoantigens, rendering it a good candidate for immunotherapy interventions. ${ }^{5}$ Besides the tumor mutational burden (TMB), in terms of tumor's T-cell infiltration head and neck cancer falls into the category of immunologically "cold" tumors, particularly the HPV- subtype. ${ }^{6}$ In comparison, some melanomas have both high TMB and are immunologically "hot" (inflamed), whereas prostate cancers typically are low-TMB/immunologically "cold" tumors.

How a head neck cancer evades immune system surveillance is an area of active research and includes a variety of reasons such as lack of neoantigens, dysfunction of antigen-presenting cells, absence of T-cell activation, presence of suppressive Tregs, impaired trafficking of activated T-cells, impaired infiltration of the tumor, expression of "don't eat me" molecules from the cancer cells such as PD-L1, etc. ${ }^{7}$ In this context, the role of radiation therapy is to transform immunologically "cold" tumors into "hot" via immunogenic cell death induction, an effect that is colloquially known as "in-situ" vaccination. ${ }^{8,9}$ 


\section{Abscopal effect}

\section{Mechanism of action}

Historically, radiotherapy was considered to act immunosuppressive presumably due to the increased radiosensitivity of lymphocytes ${ }^{10}$ and the use of large radiation fields. This concept is being challenged via the realization that ionizing radiation can induce diverse forms of cell death and dying cell clearance, whose immunological effects could be exploited for the improvement of tumor control. ${ }^{11}$

The various cell death phenotypes include apoptosis (programmed cell death), necrosis, mitotic catastrophe and senescence. ${ }^{8}$ Some of these modes, e.g. necrosis, carry an inherent immunogenic potential due to the release of danger signals and damage-associated molecular patterns (DAMP) that attract immune's system attention. In short, the release of DAMPs triggers extravasation of monocytes and stimulates the maturation of dendritic cells (DC). Activated DCs present tumor antigens to naïve T-cells to counteract both the irradiated tumor as well as distant, out-of-field metastases via circulation. ${ }^{8}$

Conversely, other dying cell modes (e.g. apoptosis) are not perceived as dangerous events and, therefore, are unable to induce immune responses. Whether an irradiated cell dies via A or B process depends on the irradiation dose and fractionation, the cellular origin, the functionality of cycle checkpoints and the overall genetic assortment of the cell. ${ }^{8}$

Current research focuses on how to optimize radiotherapy's parameters (i.e. dose, fractionation, sequencing with immune checkpoint inhibitors etc.) in order to maximize the probability of inducing abscopal effect. ${ }^{1}$ Also, there is an unmet need for reliable predictors in order to identify those patients who would most likely benefit from combined radioimmunotherapy interventions. ${ }^{12}$

\section{Optimal radiotherapy parameters}

Although it is relatively easy to observe abscopal effect in the preclinical setting, its occurrence in clinical practice remains rare. The biological effective dose (BED) appears to be a significant determinant and its relationship with response has recently been modelled. ${ }^{13}$ With high BED the probability of observing an abscopal effect is increased. Regarding the right fractionation scheme, contradicting results have been reported in the literature. ${ }^{8}$ There is evidence corroborating the use of hypofractionated schedules, such as 8 Gy x 3 , due to their potential synergy with anti-CTLA4/anti-PD1 drugs. ${ }^{14}$ Though, daily doses more than 12 Gy have been found to increase Trex1 levels resulting in a significant decrease in cytosolic dsDNA and undermining the aforementioned synergism.

Whether irradiating a single metastatic site is sufficient to induce abscopal effects is debatable. There are preclinical and clinical evidence suggesting that single site irradiation is suboptimal and that we should adopt irradiation of multiple/all lesions. ${ }^{15}$ Elective nodal irradiation of the tumor's draining lymph nodes likely attenuates the combinatorial efficacy with immune checkpoint blockade and its exclusion in future clinical trials should be examined. ${ }^{16}$ This is most likely due to reduced immune infiltration and an unfavorable balance between CD8 effector and Treg cells.

Regarding the optimal timing we may carefully extrapolate from the PACIFIC trial where patients randomized within 14 days of chemoradiation completion to maintenance with durvalumab (antiPDL1) had an HR of 0.39 (95\% CI, 0.26-0.58) compared to 0.63
(95\% CI, 0.49-0.80) when randomization occurred between 14 and 42 days. ${ }^{17}$ This differential benefit may be explained on the basis of a synergistic effect between radiation and immunotherapy or it may represent a case of selection bias (patients more fit were more likely to undergo immediate randomization after the completion of concurrent therapy $\left.{ }^{18}\right)$. Last, the status of $\mathrm{p} 53$ protein appears to play a key role, since in $\mathrm{p} 53$ knockout mice or in mice with pharmacological inhibition of p53 an abscopal antitumor response could not be observed, irrespective of the prescribed dose. ${ }^{13}$

\section{Clinical trials}

There are many ongoing clinical trials involving radiotherapy and immune checkpoint blockade for recurrent or metastatic head and neck cancer. ${ }^{19}$ NCT02684253 was a phase II trial of patients with M1 head and neck squamous cell cancer that were randomized to nivolumab monotherapy or nivolumab plus stereotactic body radiotherapy (SBRT) to a single metastatic site with 9 Gy $x$ 3. Despite its promising design it was a negative trial, since there was no difference in the objective response rates between the two arms. ${ }^{20}$

EAGLE trial studied the combined checkpoint inhibition with durvalumab and tremelimumab (anti-CTLA4) in patients with recurrent or metastatic head and neck squamous cell carcinoma who experienced disease progression following platinum-based chemotherapy, regardless of their PD-L1 tumor status. The trial was also a negative one but it did not make use of SBRT. ${ }^{21}$ We do believe that SBRT is an essential component in the context of this discussion, given the low overall response rates $(\sim 13-18 \%)$ for platinumrefractory recurrent/metastatic head and neck cancer. ${ }^{22}$

Another ongoing clinical trial from Bahig et al. ${ }^{23}$ is using double checkpoint inhibition with durvalumab plus tremelimumab and SBRT (10 Gy x 5) to $2-5$ metastatic sites. ${ }^{23}$ In our opinion, this is one of the most promising studies because it uses combined immune checkpoint blockade, a highly effective dose schedule in terms of BED and allows the irradiation of more than a single metastatic site.

\section{Conclusion}

In conclusion, abscopal effect is a rare phenomenon, particularly in the context of head neck cancer. Its frequency is increasing after the introduction of immune checkpoint inhibitors. There is an urgent need for clinical trials in order to determine optimal radiotherapy parameters and maximize abscopal's effect induction probability. At the moment, concurrent administration of ICIs, moderately hypofractionated regimens (not larger than $12 \mathrm{~Gy} / \mathrm{fx}$ ) with high BED, irradiating more than 1 metastatic focus and sparing regional draining lymph nodes represent our most educated guesses.

\section{Acknowledgments}

None.

\section{Conflicts of interest}

The authors declare there are no conflicts of interest.

\section{References}

1. Dagoglu N, Karaman S, Caglar HB, et al. Abscopal effect of radiotherapy in the immunotherapy era: systematic review of reported cases. Cureus. 2019;11(2):e4103.

2. Mole RH. Whole body irradiation; radiobiology or medicine? Br J Radiol. 1953;26(305):234-241. 
3. McCulloch HD. On the analogy between spontaneous recoveries from cancer and the specific immunity induced by $\mathrm{x}$ ray irradiations of the lymphatic glands involved. Br Med J. 1908;2(2494):1146-1148.

4. Shinde A, Novak J, Freeman ML, et al. Induction of the abscopal effect with immunotherapy and palliative radiation in metastatic head and neck squamous cell carcinoma: a case report and review of the literature. Cureus. 2019;11(3):e4201.

5. Alexandrov LB, Nik-Zainal S, Wedge DC, et al. Signatures of mutational processes in human cancer. Nature. 2013;500(7463):415-421.

6. Maleki Vareki S. High and low mutational burden tumors versus immunologically hot and cold tumors and response to immune checkpoint inhibitors. J Immunother Cancer. 2018;6(1):157.

7. Horton JD, Knochelmann HM, Day TA, et al. Immune evasion by head and neck cancer: foundations for combination therapy. Trends Cancer Res. 2019;5(4):208-232.

8. Brix N, Tiefenthaller A, Anders H, et al. Abscopal, immunological effects of radiotherapy: Narrowing the gap between clinical and preclinical experiences. Immunol Rev. 2017;280(1):249-279.

9. Bonaventura P, Shekarian T, Alcazer V, et al. Cold tumors: a therapeutic challenge for immunotherapy. Front Immunol. 2019;10:168.

10. Trowell OA. The sensitivity of lymphocytes to ionising radiation. J Patho Bacteriol. 1952;64(4):687-704.

11. Lauber K, Ernst A, Orth M, et al. Dying cell clearance and its impact on the outcome of tumor radiotherapy. Front Oncol. 2012;2:116.

12. Liu Y, Dong Y, Kong L, et al. Abscopal effect of radiotherapy combined with immune checkpoint inhibitors. J Hematol Oncol. 2018;11(1):104.

13. Marconi R, Strolin S, Bossi G, et al. A meta-analysis of the abscopal effect in preclinical models: Is the biologically effective dose a relevant physical trigger? PLoS One. 2017;12(2):e0171559.
14. Vanpouille-Box C, Alard A, Aryankalayil MJ, et al. DNA exonuclease Trex1 regulates radiotherapy-induced tumour immunogenicity. Nat Commun. 2017;8:15618.

15. Brooks ED, Chang JY. Time to abandon single-site irradiation for inducing abscopal effects. Nat Rev Clin Oncol. 2019;16(2):123-135.

16. Marciscano AE, Ghasemzadeh A, Nirschl TR, et al. Elective nodal irradiation attenuates the combinatorial efficacy of stereotactic radiation therapy and immunotherapy. Clin Cancer Res. 2018;24(20):5058-5071.

17. Antonia SJ, Villegas A, Daniel D, et al. Overall survival with durvalumab after chemoradiotherapy in stage III NSCLC. $N$ Engl J Med. 2018;379(24):2342-2350

18. Bang A, Schoenfeld JD, Sun AY. PACIFIC: shifting tides in the treatment of locally advanced non-small cell lung cancer. Transl Lung Cancer Res. 2019;8:S139-S146.

19. Manukian G, Bar-Ad V, Lu B, et al. Combining radiation and immune checkpoint blockade in the treatment of head and neck squamous cell carcinoma. Front Oncol. 2019;9:122.

20. McBride SM, Lee NY, Pfister DG, et al. Biomarker predictors of outcome from a randomized trial of nivolumab $+/$ - stereotactic body radiotherapy (SBRT) in metastatic (M1) head and neck squamous cell carcinoma (HNSCC). J Clin Orthod. 2019;37(15):6063-6063.

21. Licitra LF, Haddad RI, Even C, et al. EAGLE: A phase 3, randomized, open-label study of durvalumab (D) with or without tremelimumab (T) in patients (pts) with recurrent or metastatic head and neck squamous cell carcinoma (R/M HNSCC). J Clin Orthod. 2019;37(15):6012-6012.

22. Oliva M, Spreafico A, Taberna M, et al. Immune biomarkers of response to immune-checkpoint inhibitors in head and neck squamous cell carcinoma. Ann Oncol. 2019;30(1):57-67.

23. Bahig H, , Aubin F, Stagg J, et al. Phase I/II trial of Durvalumab plus Tremelimumab and stereotactic body radiotherapy for metastatic head and neck carcinoma. BMC Cancer. 2019;19(1):68. 